\title{
STUDY AND SIMULATION OF DC MICRO GRID TOPOLOGIES IN CASPOC
}

\author{
Wouter Aerts ${ }^{1,2}$; Simon Willems ${ }^{1,2}$; Dries Haeseldonckx ${ }^{2}$; Pepijn van Willigenburg ${ }^{3}$; Johan \\ Woudstra $^{3}$; Stijn De Jonge ${ }^{1,2}$ \\ ${ }^{1}$ CORE crba-so - Kapeldreef $75-3001$ Heverlee - Belgium \\ ${ }^{2}$ KULeuven campus GROEPT - A. Vesaliusstraat $13-3000$ Leuven - Belgium \\ ${ }^{3}$ The Hague University of Applied Sciences - Rotterdamseweg 137-2628 AL Delft - The Netherlands
}

\begin{abstract}
As the impact of our actions on the climate become more and more clear and environmental awareness is rising, the quest for increasing efficiency and lower environmental impact becomes very important. Efficiency is particularly important in the field of electricity consumption, which keeps on rising as electrification of our transportation, houses, offices and more continues worldwide. These loads and sustainable sources have one thing in common: Direct Current. To successfully respond to this growing usage of direct current (DC) systems it is important to provoke an evolution in the provision of DC infrastructure.
\end{abstract}

The goal of this paper is to create a methodology to calculate and evaluate the power losses in both traditional AC grids and DC microgrids. This is done through simulation models made by Caspoc, a software for modeling and simulating physical systems in analog/power electronics, electric power generation/conversion/distribution and mechatronics. The results are compared on the quantifiable indicator: energy savings. The impact of cable losses and different converters is calculated through the simulation.

This methodology and simulation strategy can be the basis for the optimal grid design in other infrastructures and cases. The model will be validated with intensive tests of household equipment in a later stage of the project, this paper focuses on the model and methodology itself.

\section{INTRODUCTION}

From a consumers point of view, one can see the evolution towards more and more DC usage. Many electrical components in our household are already working on DC. This becomes clear when we look to all the different electronic devices we daily use; e.g. laptops, TVs or even lighting [1], [2]. In current situations all of these DC appliances need inefficient rectifiers and power factor correction (PFC) circuits before they can be connected to our AC electricity grid. The efficiencies of these converters vary over a wide range so it is as user not always clear how much energy is lost when a certain appliance is used. On the other hand, renewable energy sources like solar panels produce $\mathrm{DC}$ voltages and the most common technologies to store energy also use DC voltages.
As local governments manage towards more energyefficiency, efforts are necessary to consume electricity as efficient as possible. It is expected that the consumption of electricity will increase more and more. Different reports predicts growths of the consumption with a value between 0.1 to $2.2 \%$ p.a. [3]. In every scenario the residential growth is expected to increase the most due to a growth of households and electrifications of our daily life.

These findings show that the quest for increasing efficiency and energy savings becomes very important. The use of more DC and implementation of DC grids becomes a reality. One can see that $\mathrm{DC}$ power is already present as HVDC in the transmission grid. Moreover, LVDC will become more important in the future [4], [5]. A DC microgrid is an electric DC grid in a house with power consumption of less than $5000 \mathrm{kWh}$ a year. This value represents an average consumption of a household in the Benelux over the course of a year [6].

The previous statements mean that it is desirable that all of the energy losses are revisited and possible solutions are studied. This paper introduces a method to calculate and evaluate the power losses in the cables of an electrical (AC or DC) grid and with the conversion losses in applications. To explain the methodology and different steps, and to retain simplicity, an example of an AC grid with only 3 different household appliances is used in this paper. When larger and more extensive grids have to be studied, using the same method will still result in a successful calculation and evaluation. First, the software will be discussed together with the parameters and the methods to obtain these. Afterwards the results from the simulation are explained and more detailed evaluations will conclude the findings.

\section{SYSTEM LOSSES}

The use of the measured data in a simulation model would give a complex and time-consuming simulation. To speed up the simulation time and to have a generative model, a methodology was created so that calculations can be done easy and fast. It is also simple to simulate new cases and different grid topologies, both AC and DC.

\subsection{CABLE LOSSES}

In this study, losses of the cable resistance are calcultate for the AC and DC grid. Fig. 1 shows the principle of the loss calculcation. These losses can be calculated as in eq. 
1 , where $I$ is the current through the cable and $R$ is the cable resistance.

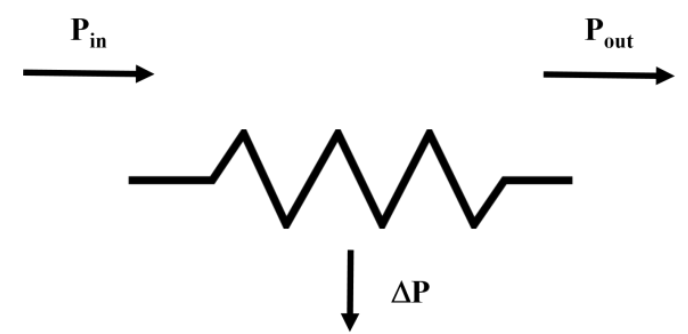

Fig. 1. Concept of the loss calculation.

$$
\Delta P=I^{2} \cdot R
$$

The model of Fig. 1 is also used in the software model to simulate and calculate the cable losses. From the consumption $P$ and the power factor $P F$ of the load, the current $I_{a c}$ that is draw in the AC grid by the load can be calculated. Knowing the current $I$, the power losses of the cable resistance can be calculated using eq. 2 and 3 .

$$
\begin{aligned}
I_{a c} & =\frac{P_{i n}}{U_{a c} \cdot P F} \\
\Delta P & =R \cdot I_{a c}{ }^{2}=R \cdot \frac{P_{i n}{ }^{2}}{U_{a c^{2}}{ }^{2} \cdot P F^{2}}=\frac{R}{P F^{2}} \cdot \frac{P_{i n}{ }^{2}}{U_{a c}{ }^{2}}
\end{aligned}
$$

In the DC grid there is only active power present, so that for the same load and cable lenght the current $I_{d c}$ and power losses $\Delta P$ can be calculated through eq. 4 and 5 .

$$
\begin{aligned}
& I_{d c}=\frac{P_{\text {in }}}{U} \\
& \Delta P=R \cdot I_{d c}{ }^{2}=R \cdot \frac{P_{\text {in }}{ }^{2}}{U_{d c}{ }^{2}}
\end{aligned}
$$

\subsection{CONVERTER LOSSES}

Next to the power losses in the cables there are also losses in every converter in the grid. These will be significantly higher but are different for each type of converter and are a function of the delivered power. If a laptop converter with a power output of $70 \mathrm{~W}$ (Pout) is taken as example the average active efficiency can be calculated by: $[0.0626 \cdot \ln ($ Pout $)]+0.622=0.88$ [7]. To know the exact profile of the efficiency in function of the output power, measurements have to be performed. When implementing a DC grid, AC/DC converters have to be replaced by $\mathrm{DC} / \mathrm{DC}$ converters who are more efficient in general. Not only the converters of electronic devices will cause a rise in efficiency but also the change of motor type will be beneficial.

\subsection{MEASUREMENTS}

To have reliable input data for the power consumption profile of different household appliances, measurements on appliances are preformed. The following variables are measured:

- $\quad$ Power profile over one cylce (W)
- Total Harmonic Distortion (THD): present harmonic distortion of every device when active.

- Displacement Power Factor (dPF): $\cos \varphi$ of every device.

From the last two measurements the power factor of the appliance can be calculated [8] through eq. 6, so that the AC cable losses from eq. 3 can be calculated. This power factor is used in the simulation software as a variable for every load.

$$
P F=\frac{d P F}{\sqrt{1+T H D^{2}}}
$$

The measurements of the three household applications present in the grid used in this paper, are shown in Fig. 2.

\section{Load profile washing machine}

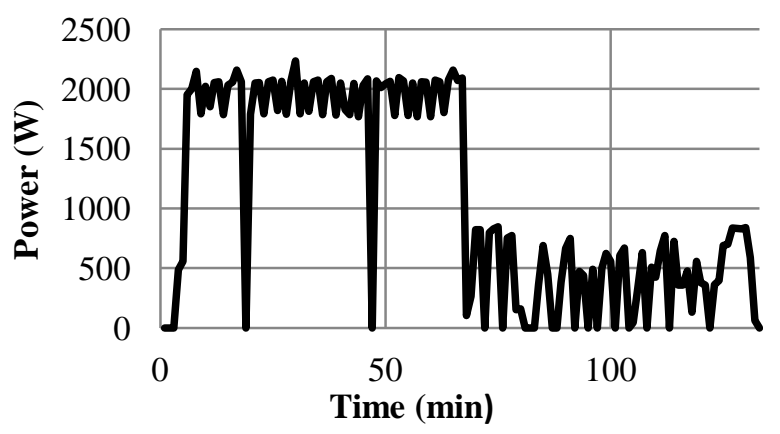

Fig. 2. Load profile of household appliance.

\section{SIMULATION OF MICROGRID}

To calculate the cable losses of the grid, the simulation software Caspoc is used. Caspoc is suited for modeling and simulating physical systems. It enables full-system virtual prototyping of applications in analog or power electronics; electric power generation, conversion, distribution and mechatronics. By using Caspoc, it is possible to model, load and control in one multilevel model [9]. In the software a simple AC model was made to represent a load, which draws a power P (Watt) from the grid and has a specific power factor PF. Fig 3 shows a small network with three different consumers:

- $\quad$ big power consumer: washing machine (load 1)

- small periodic power consumer: refrigerator (load 2)

- small power consumer with AC/DC converter: laptop (load 3).

This network is used to illustrate the methodology, which is described in the next section. For this network a cable resistance $R=0.01 \Omega / m$ is used [10] and a total cable length of $50 \mathrm{~m}$. For the AC/DC converter of the laptop an efficiency of $88 \%$ is used. 


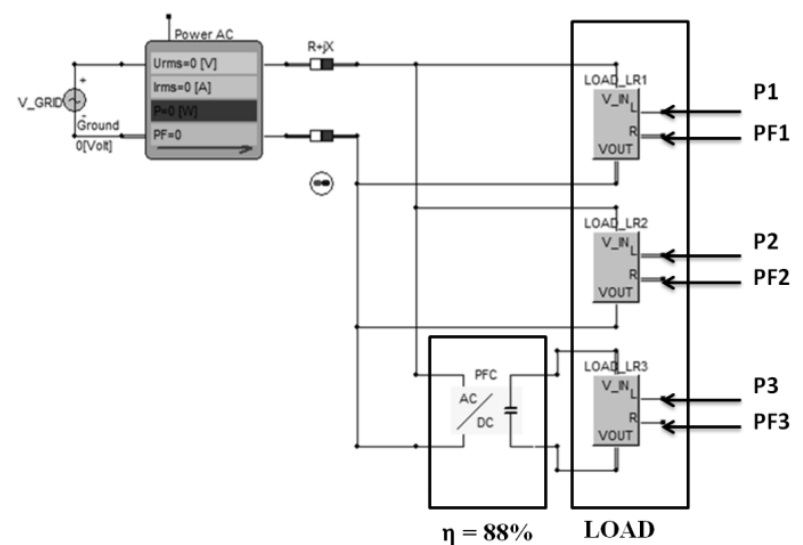

Fig. 3. Simulation network in Caspoc

\section{INFLUENCE OF APPLIANCE ON TOTAL LOSS}

It can be interesting to see what the influence of different appliances is on the losses in the cables. First of all the length of the loop where the appliance is in will affect this, but when 3 appliances are considered to be in the same loop there will still be a difference. This is due to the different amounts of power, both active as reactive, the appliance demands. This effect can be shown by calculating different distributions of a certain power level over the investigated appliances. These calculations will result in a range of power losses in the cable for the same overall power level. To show this, 3 appliances are specified: a washing machine, refrigerator and a laptop with a power factor of respectively $0.94,0.7$ and 0.82 . Now a power is distributed over the appliances on different ways as can be seen in table 1 .

Table. 1. Influence of appliance on total loss

\begin{tabular}{|c|c|c|c|c|c|}
\hline $\boldsymbol{P}(\boldsymbol{W})$ & $\begin{array}{l}\mathbf{T} 1 \\
(\boldsymbol{W})\end{array}$ & $\begin{array}{l}\mathbf{T} 2 \\
(\boldsymbol{W})\end{array}$ & $\begin{array}{c}\mathbf{T 3} \\
(\boldsymbol{W})\end{array}$ & $\begin{array}{c}\Delta \boldsymbol{P} \\
(\boldsymbol{W})\end{array}$ & $\begin{array}{c}\Delta \boldsymbol{P} \\
(\%)\end{array}$ \\
\hline 100 & 40 & 20 & 40 & 0.253 & $0.25 \%$ \\
\hline 100 & 20 & 40 & 40 & 0.285 & $0.29 \%$ \\
\hline 100 & 60 & 20 & 20 & 0.239 & $0.24 \%$ \\
\hline 100 & 40 & 40 & 20 & 0.269 & $0.27 \%$ \\
\hline 100 & 100 & 0 & 0 & 0.207 & $0.21 \%$ \\
\hline 100 & 80 & 20 & 0 & 0.227 & $0.23 \%$ \\
\hline 100 & 80 & 0 & 20 & 0.216 & $0.22 \%$ \\
\hline 100 & 0 & 100 & 0 & 0.366 & $0.37 \%$ \\
\hline 100 & 0 & 0 & 100 & 0.268 & $0.27 \%$ \\
\hline
\end{tabular}

When this data is entered in Caspoc the range of power losses in the cables can easily be achieved. The minimum is reached when the washing machine is operating at maximum power, because of the high power factor. The opposite is true for the maximum loss when the refrigerator is at his maximum power. The range of the losses is displayed in Fig. 4 over the total power consumed by the appliances. The bottom curve represents the losses in the grid when a device with high power factor is running in the grid. The top curve shows the losses when the power is consumed by appliances with low power factor. The interval between the two curves is caused when the same total power is needed for different devices in the grid with different power factors.

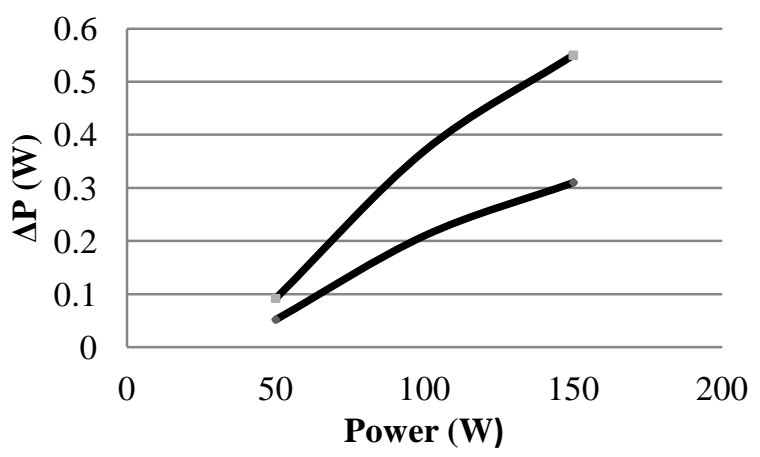

Fig. 4. Range of power losses

\section{SIMUMATION MODEL AND STRATEGY}

Step 1 - In the first step the measured data is bundled and simplified for all appliances. This is done by dividing the power consumption of the appliance in intervals, ascending from the measured minimum to the measured maximum with discrete intervals in between. Next, every interval is given a corresponding weight. This value tells how often the power consumption profile of the appliance appears within this interval. Fig. 5 shows the result for the washing machine.

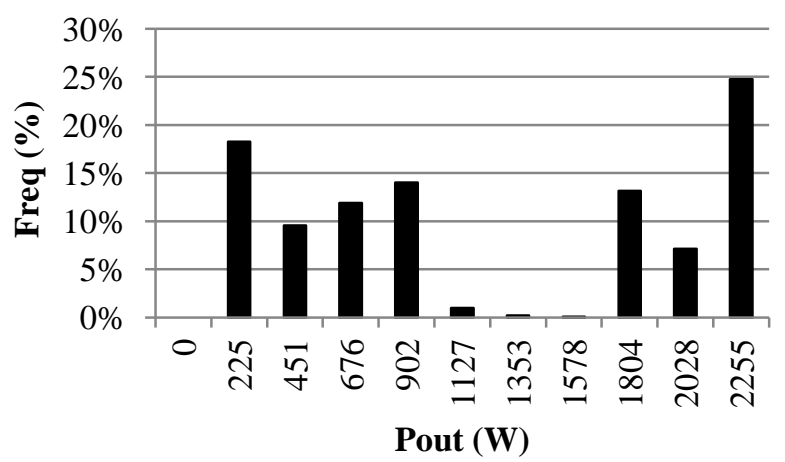

Fig. 5. Bundled data washing machine vs. frequency.

Step 2 - In the second step, the simplified data is used in the Caspoc model to calculate all the losses in the system. This is done for every appliance separately with the corresponding dataset obtained in step 1: the value of every interval is used in the Caspoc model to calculate the losses. The results show the system losses of every appliance individually in the grid, at each power consumption interval. This gives us the single effect of every device within the network, on this network.

Step 3 - Further, each calculated value obtained from the Caspoc simulation is multiplied with its corresponding weight, obtained from the first step. Next the average power loss in Watt of the appliance during one cycle is 
calculated. The results of the simulation with the values of the washing machine are shown in Table 2.

Table. 2. Simulation results.

\begin{tabular}{|c|c|c|c|c|}
\hline $\operatorname{Pin}(W)$ & $\operatorname{Pout}(W)$ & $\Delta P$ & Freq. (\%) & $\operatorname{Loss}(\boldsymbol{W})$ \\
\hline 0 & 0 & 0 & $0 \%$ & 0,00 \\
\hline 226 & 225 & 1 & $18 \%$ & 0,18 \\
\hline 457 & 451 & 5 & $10 \%$ & 0,52 \\
\hline 688 & 676 & 12 & $12 \%$ & 1,44 \\
\hline 925 & 903 & 22 & $14 \%$ & 3,09 \\
\hline 1161 & 1127 & 34 & $1 \%$ & 0,34 \\
\hline 1404 & 1353 & 51 & $0 \%$ & 0,10 \\
\hline 1646 & 1577 & 68 & $0 \%$ & 0,03 \\
\hline 1896 & 1804 & 92 & $13 \%$ & 12,03 \\
\hline 2143 & 2028 & 115 & $7 \%$ & 8,20 \\
\hline 2402 & 2256 & 146 & $25 \%$ & 36,18 \\
\hline & & \multicolumn{3}{|c|}{ Average power loss: } \\
\hline
\end{tabular}

Step 4 - The same simulation can be done for the other appliances, in this case the refrigerator and laptop. All the resulting values can be put into a database, which is given a overview of all the losses for every appliance when they are running individual in the grid.
Step 5 - At this moment the individual losses of every appliance are known. Although it is more interesting to know the total losses of the network when in a realistic situation; this means for a longer timeframe and simultaneously. To find these results, the load profiles of the applications are put into a timeline as shown in Fig. 6. Again, to simplify and illustrate the methodology a time laps of only 2 hours is used for only three appliances: a washing machine (T1), a refrigerator (T2) and a laptop (T3). The load profiles are divided into equally large time intervals $(\Delta t)$ and, if necessary the system losses are calculated at every new interval.

The following gives an example of the sequence for evaluating the timeline. At point A, only the refrigerator is turned on. Hence the value of the system losses can be obtained from the database which was created at step 4 . At the next points the same logic is applied, since no other device is turned on. However, at point B this is no longer valid because the refrigerator and the washing machine are active at the same time. This is a new situation, for which the system losses cannot be obtained from the database of step 4 . This means the power consumption of these two devices $P_{T 1 B}$ and $P_{T 2 B}$ are used in the Caspoc model as variables to calculate the system losses $\Delta P_{B}$ for the situation at point $\mathrm{B}$. This value can now be written in the database. At the next interval, point $\mathrm{C}$, only the washing machine is active in the grid, so the value of the

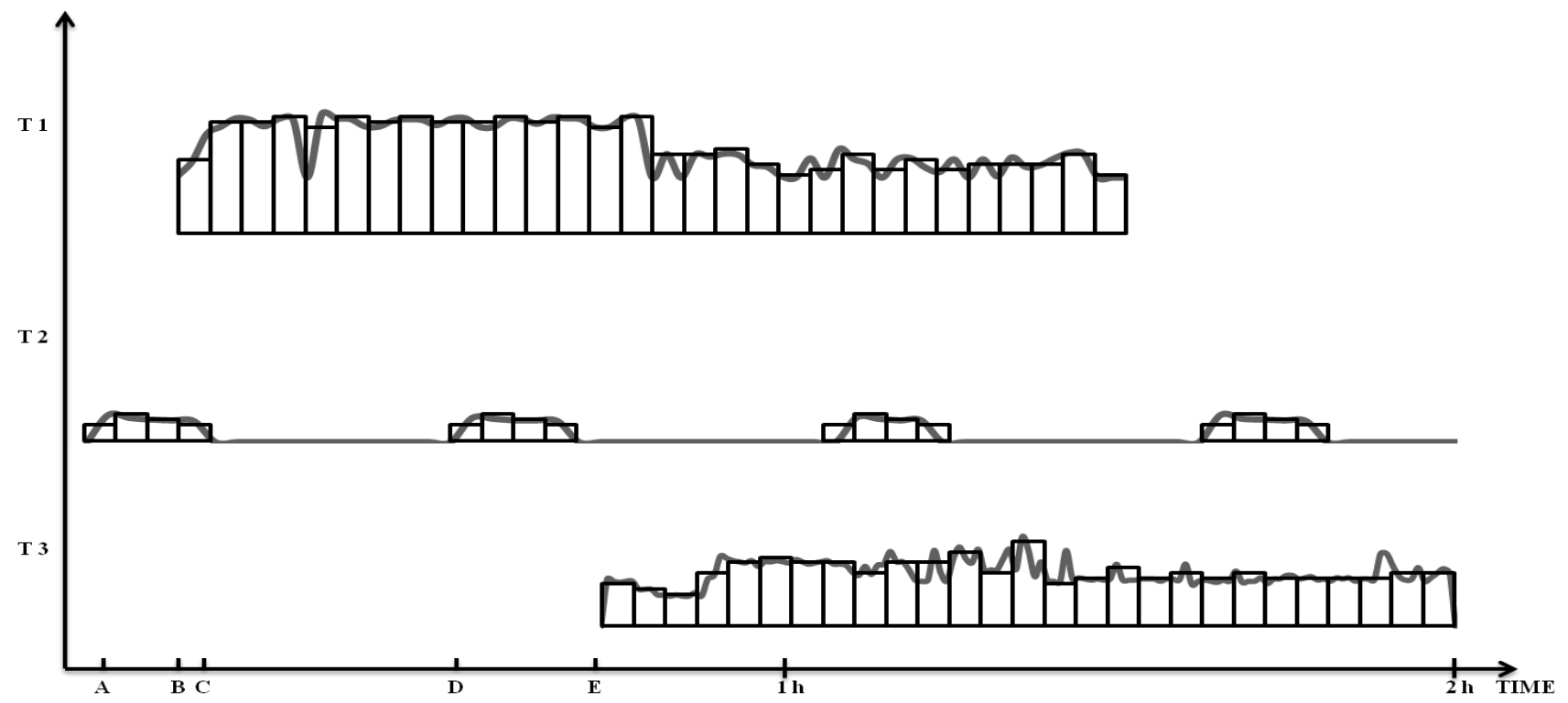

\begin{tabular}{|c|c|c|c|c|c|c|c|c|}
\hline TIME & $P_{T 1}$ & $P_{T 2}$ & $P_{T 3}$ & $P_{\text {in }}$ & $P_{\text {out }}$ & Freq. & $\Delta P$ & $\Delta W$ \\
\hline A & 0 & $P_{T 2, A}$ & 0 & $P_{i n, A}$ & $P_{\text {out }, A}$ & $y_{A}$ & $\Delta P_{A}$ & $\Delta P_{A} \cdot y_{A} \cdot \Delta t$ \\
\hline $\mathrm{B}$ & $P_{T 1, \boldsymbol{B}}$ & $P_{T 2, \boldsymbol{B}}$ & 0 & $P_{i n, B}$ & $P_{\text {out }, B}$ & $y_{B}$ & $\Delta P_{B}$ & $\Delta P_{B} \cdot y_{B} \cdot \Delta t$ \\
\hline $\mathrm{C}$ & $P_{T 1, C}$ & 0 & 0 & $P_{i n, C}$ & $P_{\text {out }, \boldsymbol{C}}$ & $y_{C}$ & $\Delta P_{C}$ & $\Delta P_{C} \cdot y_{C} \cdot \Delta t$ \\
\hline $\mathrm{D}$ & $P_{T 1, \boldsymbol{D}}$ & $P_{T 2, \boldsymbol{D}}$ & 0 & $P_{i n, \boldsymbol{D}}$ & $P_{\text {out }, \boldsymbol{D}}$ & $y_{D}$ & $\Delta P_{D}$ & $\Delta P_{D} \cdot y_{D} \cdot \Delta t$ \\
\hline $\mathrm{E}$ & $P_{T 1, E}$ & 0 & $P_{T 3, E}$ & $P_{i n, E}$ & $P_{\text {out }, E}$ & $y_{E}$ & $\Delta P_{E}$ & $\Delta P_{E} \cdot y_{E} \cdot \Delta t$ \\
\hline \multicolumn{6}{|c|}{ Fig. 6. Timeline } & $\Delta \boldsymbol{P}_{\text {Total }}$ & $=\frac{\boldsymbol{\Sigma} \Delta \mathbf{P}}{\mathbf{n}}$ & $\Delta W_{\text {Totaal }}=\Sigma \Delta W$ \\
\hline
\end{tabular}


system losses can again be obtained from the database created in step 4. At point $\mathrm{D}$ and $\mathrm{E}$ there are again multiple active devices in the network, respectively refrigerator and washing machine and washing machine and laptop. This method can be repeated for all the intervals, for the full length of the timeline. Every time a similar situation occurs, the frequency column in the table of Fig. 6 can be increased by 1 . By calculating the losses for each time interval of the cycle, the total energy loss $\Delta W_{\text {Totaal }}$ of this grid during the 2 hour cycle can be calculated.

\section{RESULTS AND CONCLUSION}

The results of the grid model discused in the previous section are shown in tabel 3.

\begin{tabular}{|c|c|c|c|c|}
\hline TIME & $\boldsymbol{P}_{\text {in }}$ & $\boldsymbol{P}_{\text {out }}$ & $\Delta \boldsymbol{P}$ & $\Delta \boldsymbol{W}$ \\
\hline $\mathrm{A}$ & 35 & 35 & 0 & 0,00 \\
\hline $\mathrm{B}$ & 102 & 102 & 0 & 0,00 \\
\hline $\mathrm{C}$ & 1520 & 1518 & 2 & 0,05 \\
\hline $\mathrm{D}$ & 2036 & 2001 & 35 & 0,88 \\
\hline $\mathrm{E}$ & 1817 & 1766 & 51 & 1,28 \\
\hline $\mathrm{n}$ & $P_{\text {in }, n}$ & $P_{\text {out }, n}$ & $\Delta P_{n}$ & $\Delta W_{n}$ \\
\hline
\end{tabular}

Table. 3. Results of grid simulation

The total energy loss of the grid during the time cycle of 2 hours can be calculated to be $\Delta W_{\text {Totaal }}=\Sigma \Delta W=$ $13,21 \mathrm{Wh}$. The method discussed in this paper presents and easy and fast way to calculate the grid losses. When the grid is expanded or new devices are added, only the intervals with unknown values need to be calculated in the Caspoc model, which reduces the simulation time. The method, and the combination with Caspoc offers a reliable tool to calculate and compare losses of different grids (both $\mathrm{AC}$ and DC) and topologies.

So far the research of this paper was limited to an AC grid with a few defined loads. The same methodology can be used to achieve results for other cases and compare them with the original. In the future a full $\mathrm{AC}$ grid will be compared with DC microgrids with a supply voltage of respectively 230,350 and 380 VDC. These voltages are chosen because of the frequent occurrence in literature. In this case $\mathrm{a} \pm 350 \mathrm{VDC}$ is looking the most promising, but still needs confirmation through research. Selecting \pm 350 VDC in a micro grid could give the following benefits [6]:

- The voltage level gives an appropriate power density and an optimal usage of copper.

- Safe and efficient voltage for domestic devices using the +350 VDC line.

- High power connections possible by using a \pm 350 VDC line for dedicated purposes like charging EVs.
A comparison will be made between the cable and convertor losses in the different cases. Further the reduction of the cross section of the cables in de DC microgrids will be studied, which can lead to savings in the total amount of used copper. As last parameter a redesign of the loops in the grid can also give a reduction in the amount of copper. An example here is a grid with 2 loops, one with the 0 and +350 VDC cable and one with the 0 and -350 VDC cable. Here is also the possibility to make a 700 VDC connection with the -350 and +350 VDC cable for high power consuming applications

\section{REFERENCES}

[1] George, K.: "DC Power production, Delivery and Utilization." Electric Power research Institute, June 2006 (White paper).

[2] Galvin Electricity Initiative. The Galvin Path to Perfect Power - A Technical Assessment. Galvin Electricity Initiative, 2007.

[3] Climact. Electrical energy savings scenarios for Belgium. February 2012.

[4] Woudstra, JB, van Willigenburg, P, Groenewald, BBJ, Stokman, H, De Jonge, S ,Willems, S: "An Introduction to Direct Current Distribution Grids." (2013)

[5] Carlsson, L, Flisberg, G, Weimers, L. "Recent evolution in classic HVDC". The 4th International Conference on Power Transmission \& Distribution Technology, 2003, Changsha, PR China.

[6] Willems, S, Aerts, W, De Jonge, S, Haeseldonckx, D, van Willigenburg, P, Woudtra, JB, Stokman, H: "Sustainalbe Impact and Standardization of a DC Micro Grid." EcoDesing 2013 International Symposium, 2013.

[7] Energystar. [Accessed 20 February 2014]. http://www.energystar.gov/ia/partners/prod_develop ment/revisions/downloads/International_Efficiency_ Marking_Protocol.pdf

[8] Putteman, K, Ryckaert, W: "Wat betekent Power Factor?" Groen Licht Vlaanderen: energiebesparing met beter licht, KAHO, Gent, 2008.

[9] Integrated. [Accessed 20 February 2014] . Copyright policy. http://www.integratedsoft.com/Products/Caspoc

[10] Cutnell, J, Johnson, K. Physics 4th edition. New York: Wiley. 1998: pp. 755. 


\section{AUTHORS}

Principal Author: Wouter Aerts is a master student


electromechanical engineering at $\mathrm{KU}$ Leuven - Campus GROUP $\mathrm{T}$ in Belgium. He combines his master studies with a postgraduate in Entrepreneurial Engineering Experience. In this program he participates in the CORE cvba-so. For his master thesis he is involved in the study and development of DC microgrids.

Principal Author: Simon Willems is a master student

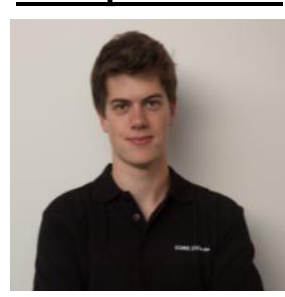
electromechanical engineering at $\mathrm{KU}$ Leuven - Campus GROUP $\mathrm{T}$ in Belgium. He combines his master studies with a postgraduate in Entrepreneurial Engineering Experience. In this program he participates in the CORE cvba-so where he is involved in the development of a DC grid for student residences.

\section{Co-author: Dries Haeseldonckx}

Co-author: $\quad$ Pepijn van Willigenbrug

Co-author: Johan Woudtra

Co-author: $\quad$ Stijn De Jonge

Presenter: $\quad$ The paper is presented by Wouter Aerts. 